\title{
Calcium Alternans is Due to an Order-Disorder Phase Transition in Cardiac Cells
}

\author{
Enrique Alvarez-Lacalle, ${ }^{1}$ Blas Echebarria, ${ }^{1}$ Jon Spalding, ${ }^{2}$ and Yohannes Shiferaw ${ }^{2}$ \\ ${ }^{1}$ Departament de Física Aplicada, Universitat Politècnica de Catalunya.BarcelonaTech, 08028 Barcelona, Spain \\ ${ }^{2}$ Department of Physics and Astronomy, California State University, Northridge, California 91330, USA
}

(Received 23 July 2014; revised manuscript received 18 October 2014; published 12 March 2015)

\begin{abstract}
Electromechanical alternans is a beat-to-beat alternation in the strength of contraction of a cardiac cell, which can be caused by an instability of calcium cycling. Using a distributed model of subcellular calcium we show that alternans occurs via an order-disorder phase transition which exhibits critical slowing down and a diverging correlation length. We apply finite size scaling along with a mapping to a stochastic coupled map model, to show that this transition in two dimensions is characterized by critical exponents consistent with the Ising universality class. These findings highlight the important role of cooperativity in biological cells, and suggest novel approaches to investigate the onset of the alternans instability in the heart.
\end{abstract}

DOI: 10.1103/PhysRevLett.114.108101

PACS numbers: 87.19.Hh, 05.70.Fh, 87.16.Xa

When a cardiac myocyte is rapidly paced by a periodic stimulus it is known to exhibit complex dynamics of both cell membrane potential and intracellular calcium (Ca). A common transition is from a periodic response to alternans, where the duration of the action potential (AP) and the $\mathrm{Ca}$ transient alternates from one beat to the next [1-3]. This phenomenon has been studied extensively since it is known that patients diagnosed with $T$-wave alternans, the resulting beat-to-beat alternation in the shape of the electrocardiogram (ECG), present a higher risk of suffering life-threatening arrhythmias [3,4]. Earlier work showed that alternans could arise from a period-doubling bifurcation associated with the kinetics of ion channels regulating the membrane potential [3]. However, experimental studies revealed that alternans may also appear due to an instability in intracellular $\mathrm{Ca}$, independently of the dynamics of voltage [5]. Global deterministic models of the average subcellular $\mathrm{Ca}$ concentration, also explained alternans as a consequence of a period doubling bifurcation in the dynamics of Ca cycling [6]. These studies overlooked, however, the stochastic nature of $\mathrm{Ca}$ signaling at the ion channel level.

Recently, several authors have studied the spatiotemporal dynamics of subcellular Ca during alternans [7-9]. In particular, Restrepo et al. [7] developed a detailed model of the three dimensional distribution of $\mathrm{Ca}$ signaling and showed that rapid pacing induced $\mathrm{Ca}$ alternans. This finding suggested that nonlinearities in the kinetics of $\mathrm{Ca}$ signaling proteins might be the underlying cause for alternans. In an important study, Rovetti et al. [10] developed a comprehensive theory of $\mathrm{Ca}$ alternans referred to as the "3R theory," which attributed alternans to the combination of the underlying stochasticity of $\mathrm{Ca}$ regulation, the regenerative recruitment of $\mathrm{Ca}$ sparks due to nearest neighbor interactions, and refractoriness in the $\mathrm{Ca}$ response. This result is important since it demonstrated that $\mathrm{Ca}$ alternans can be a direct consequence of the collective behavior of local stochastic units.
In this Letter we use a detailed model of stochastic $\mathrm{Ca}$ cycling to show that the transition from regular $\mathrm{Ca}$ cycling to alternans occurs via an order-disorder phase transition consistent with the Ising universality class. We demonstrate that this transition follows from known features of the $\mathrm{Ca}$ cycling architecture, such as the close positioning of signaling channels in microdomains, and the coupling between signaling units due to Ca diffusion. In particular, we demonstrate that these far-from-equilibrium biological systems exhibit features that can be described using equilibrium statistical mechanics, where stochasticity at the ion channel level serves the role of temperature, while cellular diffusion leads to spatial cooperativity.

Phase transition in a detailed model of Ca cycling.-The $\mathrm{Ca}$ signaling architecture [Fig. 1(a)] is designed so that changes in membrane voltage can initiate signal transduction inside the cell. A cardiac cell consists of thousands of similar domains, referred to as $\mathrm{Ca}$ release units (CaRUs), where voltage gated $\mathrm{Ca}$ channels (LCC) induce Ca release due to a nearby cluster of Ryanodine receptor (RyR) channels which control the flow of $\mathrm{Ca}$ sequestered within the sarcoplasmic reticulum (SR). RyR channels open in a $\mathrm{Ca}$ dependent manner [11] and this property ensures that a LCC channel opening in the immediate vicinity induces an autocatalytic release of $\mathrm{Ca}$ from the RyR cluster, referred to as a $\mathrm{Ca}$ spark [11]. Under normal conditions several thousand sparks are triggered in the cell and the Ca released summates to form the global Ca transient [12].

To explore the collective dynamics of these signaling domains we first note that CaRUs in ventricular myocytes are distributed along planes perpendicular to the longitudinal axis of the cell, called $Z$ planes. Each $Z$ plane is effectively a two-dimensional (2D) network of CaRUs. We therefore study the dynamics of Ca cycling in an $L \times L$ array of signaling units [Fig. 1(b); full details of the model are given in the Supplemental Material [13]]. The local signal transduction is captured via a direct simulation 


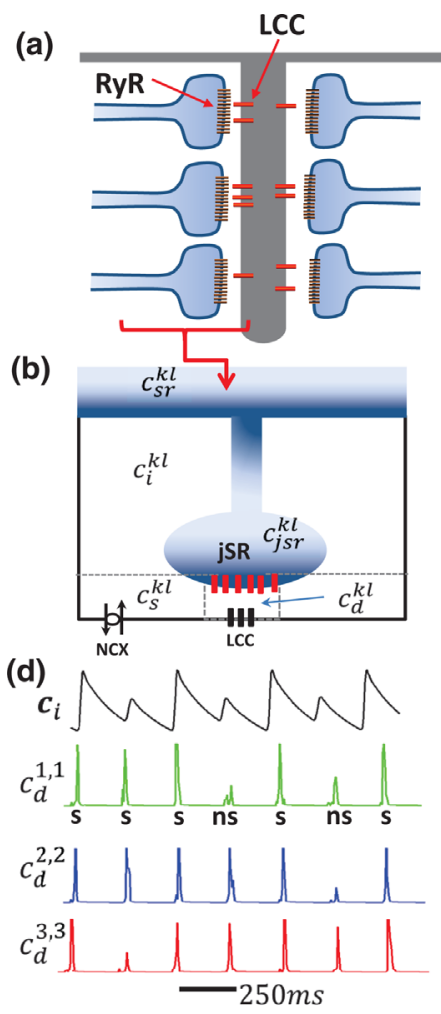

sarcolemma

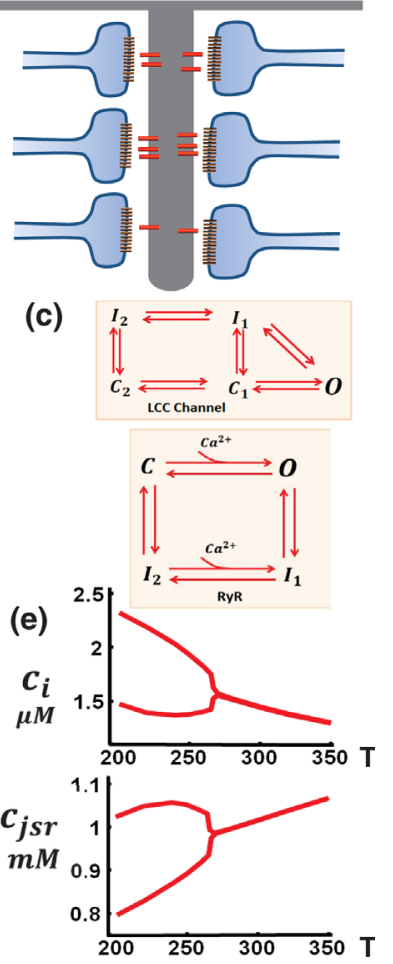

FIG. 1 (color online). Ca signaling architecture in cardiac myocytes. (a) Two-dimensional model of diffusively coupled compartments. LCC channels $(\sim 1-5)$ deliver $\mathrm{Ca}$ to the dyadic space that contains a cluster ( 10-150) of RyR channels. (b) Each $\mathrm{Ca}$ release unit (CaRU) is composed of 5 compartments: the dyadic junction with $\mathrm{Ca}$ concentration $c_{d}$; the submembrane space $\left(c_{s}\right)$; the bulk myoplasm $\left(c_{i}\right)$; the junctional SR (jSR) with concentration $\left(c_{\mathrm{jsr}}\right)$; and the network SR $\left(c_{s r}\right)$. (c) Markovian models of the LCC and RyR channels. (d) Average cytosolic Ca $\left(c_{i}\right)$ displaying $\mathrm{Ca}$ alternans at a pacing rate of $250 \mathrm{~ms}$, along with representative traces of $c_{d}$. (e) Bifurcation of the whole cell average $\mathrm{Ca} c_{i}$ and the junctional SR load $c_{\mathrm{jsr}}$.

of 1-5 LCC channels in close proximity to 10-100 RyR channels. Here, each channel is modeled by simulating the stochastic channel transitions between states of experimentally derived Markovian schemes [Fig. 1(c) and [13]]. These junctions are coupled via diffusive currents in the cytosol and SR. Then, we pace the array of signaling units using an AP clamp. Indeed, we find that when our system is paced at a fast cycle length $(T)$ then the $\mathrm{Ca}$ transient averaged over all units exhibits an alternating beat-to-beat sequence. In Fig. 1(d) we show a representative trace of the whole cell average $\mathrm{Ca}$ transient, defined as $c_{i}=\left(1 / L^{2}\right) \sum_{k l} c_{i}^{k l}$, demonstrating Ca transient alternans similar to that observed experimentally [5]. We also plot the local $\mathrm{Ca}$ concentration within the dyadic junction $c_{d}^{k l}$ for three representative compartments, showing that the local response is stochastic and can exhibit stereotypical $\mathrm{Ca}$ spark events (S), or nonsparking events (NS) where the local autocatalytic release fails or is abbreviated.

In Fig. 1(e) we show the alternans bifurcation of the steady state average peak $\mathrm{Ca}$ transient (and jSR concentration) as a function of pacing rate $T$.

To describe the transition to alternans in a cardiac cell we first define a local order parameter that gives a measure of the phase of alternans in different parts of the cell. Inspired by previous work on the analysis of phase transitions in chaotic coupled map lattices [22], we use

$$
\begin{aligned}
m_{k l}(n) & =\operatorname{sgn}\left\{(-1)^{n}\left[c_{\mathrm{jsr}}^{k l}(n)-c_{\mathrm{jsr}}^{k l}(n-1)\right]\right\} \\
& =\operatorname{sgn}\left[(-1)^{n} \Delta c_{\mathrm{jsr}}^{k l}(n)\right],
\end{aligned}
$$

where $\operatorname{sgn}[x]$ refers to the sign of the argument $x$, $c_{\mathrm{jsr}}^{k l}(n)$ denotes the jSR load at site $(k, l)$, at the beginning of the $n$th beat, and where the $(-1)^{n}$ is included so that the sign of $m_{i j}(n)$ gives a measure of the phase of alternans; i.e., $m_{k l}=+1$ for all $n$ denotes a large-small-large-small sequence of $c_{\mathrm{jsr}}^{k l}(n)$. Global alternans appears when the concentrations in $c_{\mathrm{jsr}}^{k l}$ alternate from beat to beat $\left[\Delta c_{\mathrm{jsr}}^{k l}(n) \neq 0\right]$ with the same overall phase. Indeed, the magnitude of whole cell alternans is the system average $m(n)=\left(1 / L^{2}\right) \sum_{i j} m_{i j}(n)$, which gives a measure of the global alternans phase at beat $n$. We find that close to the transition to global alternans our order parameter displays features indicative of long-range order. In Fig. 2(a) we plot a snapshot of the steady state spatial distribution of alternans amplitude $(-1)^{n} \Delta c_{\mathrm{jsr}}^{k l}(n)$, and phase $m_{k l}(n)$, for decreasing pacing rates. We observe that global alternans forms via the gradual coalescence of in-phase domains similar to the patterns observed in the 2D Ising model close to the critical temperature. A plot of the time dependence of the average alternans phase [Fig. 2(b)] displays large temporal fluctuations near the critical pacing rate, which is indicative of critical slowing down near a second order phase transition. This result is also consistent with the observation of a diverging correlation length [Fig. 2(c)] as the critical pacing rate is approached.

Finite size scaling analysis.-To uncover the nature of the transition we have computed moments of the alternans phase defined as

$$
\left\langle m^{k}\right\rangle=\frac{1}{N} \sum_{n=1}^{N}|m(n)|^{k},
$$

where $N$ is the number of beats. In Fig. 2(c) we plot $\left\langle m^{2}\right\rangle$ vs the pacing rate $T$, showing the gradual onset of alternans. In the range of pacing intervals where $\left\langle m^{2}\right\rangle$ begins to increase, the susceptibility, defined as $\chi=L^{2}\left(\left\langle m^{2}\right\rangle-\right.$ $\left.\langle m\rangle^{2}\right)$, increases with system size [Fig. 2(c)], suggesting that it diverges in the thermodynamic limit, which is indicative of long-range correlations near a phase transition. To find the critical pacing period $T_{c}$ we compute the Binder cumulant [23] $U=-3+\left\langle m^{4}\right\rangle /\left\langle m^{2}\right\rangle^{2}$, which is 

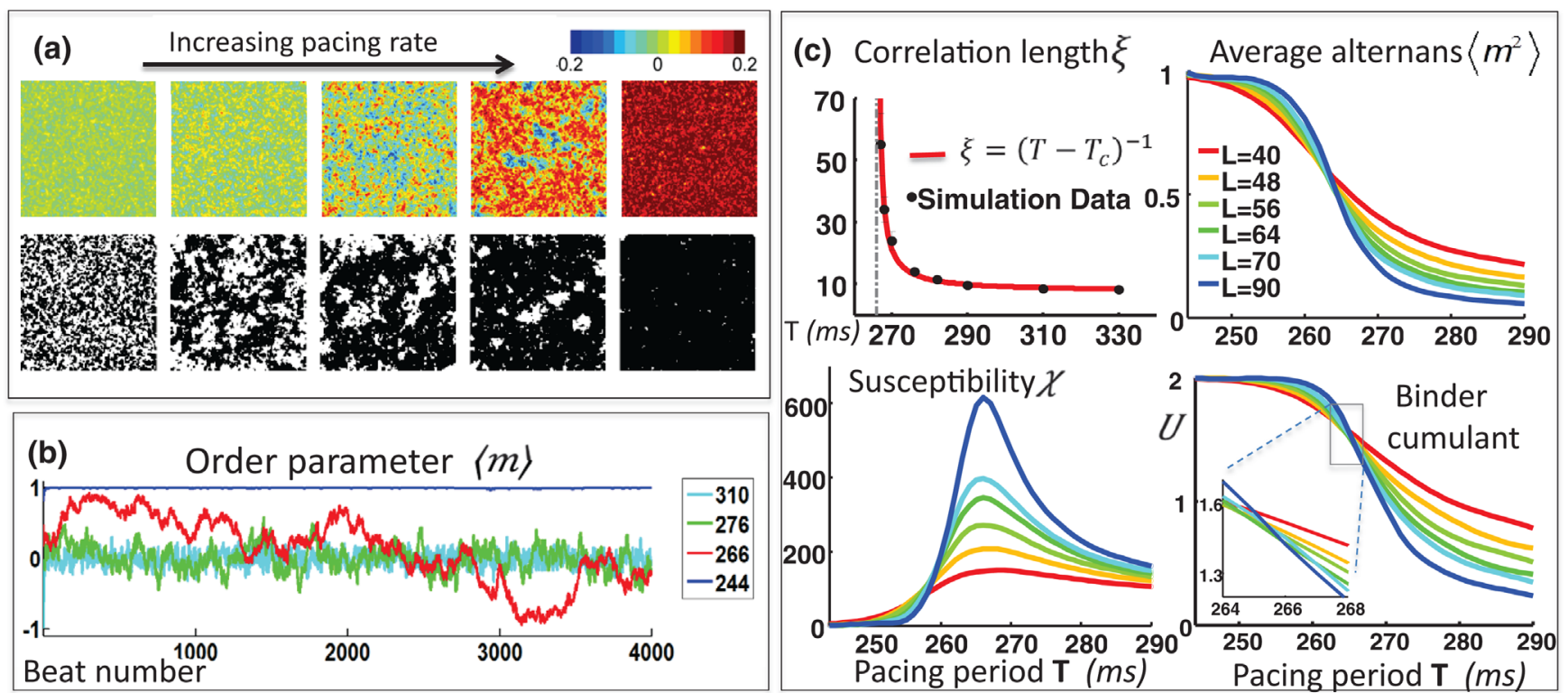

FIG. 2 (color online). (a) Snapshots of the spatial distribution of the alternation of the jSR load (top, beat-to-beat difference in mM encoded in color) and alternans phase $m_{k l}$ (bottom), for $L=1000$, as the pacing period is decreased, showing a transition from a disordered to an ordered phase. (b) Alternans phase as a function of beat number for system size $L=200$. (c) Correlation length (in units of number of release sites) as a function of pacing period. A fit of the data gives an exponent $\nu=1$, consistent with an Ising scaling. Pacing rate dependence of $\left\langle m^{2}\right\rangle$ and susceptibility $\chi$ for several system sizes. Binder cumulant curves for several system sizes showing an intersection at a critical pacing rate $T_{c} \approx 266 \mathrm{~ms}$.

independent of system size according to the finite size scaling ansatz $\left\langle m^{k}\right\rangle=L^{-k \beta / \gamma} F\left[\left(T-T_{c}\right) L^{1 / \nu}\right]$. Numerical simulations of systems sizes $40<L<90$, with temporal averages of $N \sim 10^{6}$ beats, reveal that the curves of the Binder cumulant intersect over a narrow range of the pacing period $T$ consistent with finite size scaling [Fig. 2(c), and inset]. At criticality, finite size scaling predicts that $\langle m\rangle \sim L^{-\beta / \nu}, \quad \chi \sim L^{\gamma / \nu}$, and $[\partial U / \partial T]_{T_{c}} \sim L^{1 / \nu}$, where $\beta, \gamma, \nu$ are critical exponents that characterize the universality class of the phase transition. Numerical simulation of these exponents gives (see full details in [13]) $\beta / \nu=$ $0.18 \pm 0.11, \quad \gamma / \nu=1.75 \pm 0.08, \quad \nu=0.91 \pm 0.14$. The values of these exponents indicate that the onset of calcium alternans is consistent with a two-dimensional orderdisorder Ising transition $(\beta / \nu=0.125, \gamma / \nu=1.75, \nu=1)$.

To check the robustness of our results we have also considered the effect of Gaussian fluctuations, up to $20 \%$ of the average, in the diffusion coefficient linking intracellular compartments. We find that disorder does not introduce significant changes in the behavior of the order parameter, showing, as in the pure system, a clear signature of a phase transition (see Supplemental Material [13]). Another important question to address is whether the order-disorder transition proposed here is sufficient to explain the formation of whole cell alternans in a 3D cardiac cell. To answer this question we have also simulated a 3D array of CaRUs, which more closely represent the internal geometry of a cardiac cell (see Supplemental Material [13] for full details). We account for the larger spacing between $Z$ planes by including a diffusive coupling between compartments in different planes that is $10 \%-20 \%$ that within a $Z$ plane. Our simulations show that this small diffusive coupling between planes is sufficient to synchronize alternans across the whole cell. Thus, the order-disorder transition reported here also occurs in a realistic 3D geometry of a cardiac cell.

Stochastic coupled maps model.-To uncover the underlying mechanism for the alternans transition we have also developed a coupled maps model of Ca cycling that mimics the key features of our detailed stochastic model. We first note that local signal transduction can be characterized by the probability, denoted as $P_{s}$, that a local LCC channel opening will trigger a $\mathrm{Ca}$ spark. To shed light on this quantity we have computed $P_{s}$ using the previous detailed model. In Fig. 3(a) we plot the probability of inducing a $\mathrm{Ca}$ spark as a function of the SR load for different numbers of RyR channels in the cluster. Our results reveal that $P_{s}$ has a robust sigmoid dependence on the SR load, which arises due to the cooperativity of RyR channels in the cluster which transition to the open state in a $\mathrm{Ca}$ dependent manner. To analyze the dynamical consequences of this feature we construct an iterative map describing Ca cycling. Here, we keep track of the beat-to-beat changes of the SR load at beat $n$, and site $i, j$, using a discrete variable $x_{i j}(n)$. The main features of our coupled map system, illustrated in Fig. 3(b), are (i) The release phase. Here, the CaRU at beat $n$ releases Ca with a trigger probability $P_{s}\left[x_{i j}(n)\right]$, which is simply the SR load dependent probability of triggering a $\mathrm{Ca}$ spark. Thus, we have 


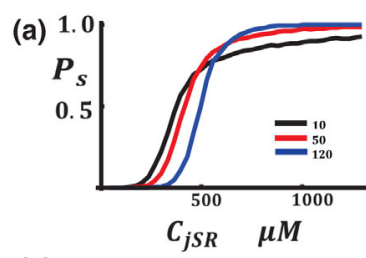

(c)
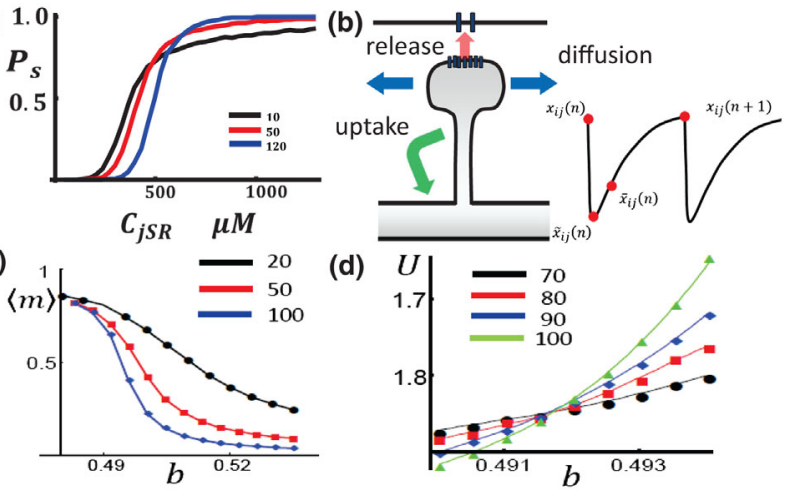

(d)

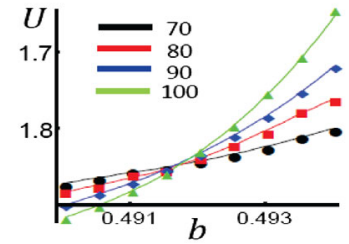

FIG. 3 (color online). (a) The probability of sparking $P_{s}$ in response to a rectangular voltage clamp from -85 to $0 \mathrm{mV}$. LCC channel openings are induced at time $t=0$ by a rapid depolarization from the resting potential. Ca spark has been triggered if the number of open RyR channels exceeds $80 \%$ of the total number of RyR channels in the cluster. (b) Illustration of a coupled maps model. Each CaRU in the cell is modeled using an iterative map of the SR concentration $x_{i j}(n)$, at site $i, j$ and beat $n$. (c) The average alternans amplitude $\langle m\rangle$ as a function of $b$ for the indicated system sizes. Model parameters used are $x^{*}=0.7$, $r=0.7$, and $\gamma=50$. (d) Binder cumulant for four system sizes showing intersection.

$$
\tilde{x}_{i j}(n)=x_{i j}(n)-r x_{i j}(n) \eta_{i j}(n),
$$

where $\tilde{x}_{i j}(n)$ is the SR load after release at the $n$th beat [Fig. 3(b)], and where $\eta_{i j}(n)$ is a random variable that is generated at each site according to the rule

$$
\eta_{i j}(n)= \begin{cases}1 & \text { with probability } P_{s}\left[x_{i j}(n)\right] \\ 0 & \text { with probability } 1-P_{s}\left[x_{i j}(n)\right]\end{cases}
$$

Here we use a probability of sparking given by $P_{s}(x)=$ $1 /\left[1+\left(x^{*} / x\right)^{\gamma}\right]$ in order to mimic the sigmoid response observed in the fully stochastic simulation. (ii) Nearest neighbor equilibration. In the next phase we mimic $\mathrm{Ca}$ diffusion between signaling units by averaging the local SR load. Therefore, we average over the nine nearest neighbors

$$
\bar{x}_{i j}(n)=\frac{1}{9} \sum_{\langle k l\rangle} \tilde{x}_{k l}(n) .
$$

(iii) SR recovery phase. The Ca released into the cell is then pumped back into the SR via SERCA uptake pumps. This effect can be modeled as

$$
x_{i j}(n+1)=\bar{x}_{i j}(n)+F\left[\bar{x}_{i j}(n)\right],
$$

where the change in SR concentration due to the uptake flux is taken to have the form $F(x)=b(1-x)$ for $x \leq 1$ and $F(x)=0$ for $x>1$. The parameter $b$ gives a measure of the amount of Ca pumped back into the SR during that beat, which is proportional to the pacing period $T$. Thus, we vary the parameter $b$ to simulate the system response to variable pacing rates.

Simulations of an $L \times L$ array of coupled maps systems reveal that at rapid pacing rates the global average $x(n)=$ $(1 / M) \sum_{i, j=1}^{M} x_{i j}(n)$ undergoes an alternans transition similar to that observed in the full stochastic simulation. To characterize the alternans transition we follow our previous approach and compute the order parameter at beat $n$ and site $i, j$ as $m_{i j}(n)=\operatorname{sgn}\left\{(-1)^{n}\left[x_{i j}(n)-x_{i j}(n-1)\right]\right\}$. In Fig. 3(c) we plot $m$ as a function of $b$ for different system sizes $L$. We observe that the average amplitude increases smoothly as the parameter $b$ is decreased below a critical point $b_{c}$, which indicates the gradual formation of an ordered phase. Furthermore, our data [Fig. 3(d)] reveal that the Binder cumulants intersect over a narrow range of $b$, which is consistent with the predictions of finite size scaling near a phase transition. We have computed the associated critical exponents using averages over $10^{9}$ beats (see [13] for details), and find exponents $\beta / \nu, \gamma / \nu$, and $\nu$ consistent with the Ising universality class.

Discussion. - In this Letter we have applied a detailed Ca cycling model to show that the transition to alternans in cardiac myocytes can be described as a second order phase transition in the Ising universality class. The mechanism for alternans is due to the fact that local $\mathrm{Ca}$ release is probabilistic and all-or-none, so that at rapid pacing rates the switchlike behavior of the probability of sparking $P_{s}$ favors alternating sequences of release and no-release. This sequence of release events is stochastic and eventually fluctuations desynchronize neighboring units. For a sufficiently steep curve, however, fluctuations are smaller and alternating sequences persist for a large number of beats, so nearest-neighbor diffusion suffices to synchronize sequences that are in phase. We find that this synchronization of local alternating sequences occurs via an order-disorder transition in the Ising universality class. This result can be partially explained by the observation that a \pm 1 alternans phase differs only by a shift of one beat, and therefore should be dynamically equivalent. This underlying Ising symmetry of the system (first pointed out in [8]), combined with short range diffusive coupling, is likely the key factor that places the system in the Ising universality class.

Critical behavior has also been observed in subcellular $\mathrm{Ca}$ dynamics in the absence of pacing. In particular, Nivala et al. [24] demonstrated that Ca released in the cell can be tuned to exhibit a self-organized critical state (SOC) in which stochastic $\mathrm{Ca}$ sparks generate $\mathrm{Ca}$ spark clusters of different sizes via propagating $\mathrm{Ca}$ waves. This critical behavior is distinct from the order-disorder transition reported here which occurs in response to pacing. In both cases $\mathrm{Ca}$ diffusion plays a crucial role. For the Ising transition $\mathrm{Ca}$ diffusion is required to synchronize local alternating responses, while the SOC state requires diffusion to induce propagating $\mathrm{Ca}$ waves in the cell. 
These results reveal that $\mathrm{Ca}$ signaling in cardiac myocytes exhibits a broad range of distinct critical behavior.

The emergence of equilibrium properties in nonequilibrium systems has also been observed in coupled chaotic maps which exhibit phase transitions [22,25], where it has been shown that a course grained description satisfies the conditions for equilibrium statistical mechanics, such as detailed balance and Gibbs distributions [26]. Our results suggest that this scenario explains the phase transition towards $\mathrm{Ca}$ alternans, where the probabilistic response of RyR and LCC channels, which determines the strength of local fluctuations, plays the role of temperature. It is the interplay between this local stochasticity and diffusive coupling that is responsible for the rich spatiotemporal structures that exhibit critical behavior.

This work was supported by the National Heart, Lung, and Blood Institute Grant No. RO1HL119095. E. A-L. and B. E. acknowledge funding from Ministerio de Ciencia e Innovación (Spain), under Grant No. FIS2011-28820C02-01.

[1] W. T. Clusin, Crit. Rev. Clin. Lab. Sci. 40, 337 (2003).

[2] J. N. Weiss, A. Karma, Y. Shiferaw, P. S. Chen, A. Garfinkel, and Z. Qu, Circ. Res. 98, 1244 (2006).

[3] J. N. Weiss, Z. Qu, P. S. Chen, S. F. Lin, H. S. Karagueuzian, H. Hayashi, A. Garfinkel, and A. Karma, Circulation 112, 1232 (2005).

[4] G. Amit, D. S. Rosenbaum, D. M. Super, and O. Costantini, Heart Rhythm 7, 763 (2010).

[5] E. Chudin, J. Goldhaber, A. Garfinkel, J. Weiss, and B. Kogan, Biophys. J. 77, 2930 (1999).

[6] Y. Shiferaw, M. A. Watanabe, A. Garfinkel, J. N. Weiss, and A. Karma, Biophys. J. 85, 3666 (2003).

[7] J. G. Restrepo, J. N. Weiss, and A. Karma, Biophys. J. 95, 3767 (2008).
[8] J. G. Restrepo and A. Karma, Chaos 19, 037115 (2009).

[9] Z. Qu, M. Nivala, and J. N. Weiss, J. Mol. Cell Cardiol. 58, 100 (2013).

[10] R. Rovetti, X. Cui, A. Garfinkel, J. N. Weiss, and Z. Qu, Circ. Res. 106, 1582 (2010).

[11] H. Cheng and W. J. Lederer, Physiol. Rev. 88, 1491 (2008).

[12] M. J. Berridge, Cell Calcium 40, 405 (2006).

[13] See Supplemental Material at http://link.aps.org/ supplemental/10.1103/PhysRevLett.114.108101, which includes Refs. [14-21], for details on the intracellular calcium cycling model and the calculation of the critical exponents, as well as the extension of our results to different geometries to support their generality.

[14] D. Sato, Y. Shiferaw, A. Garfinkel, J. N. Weiss, Z. Qu, and A. Karma, Circ. Res. 99, 520 (2006).

[15] A. Mahajan, Y. Shiferaw, D. Sato, A. Baher, R. Olcese, L. H. Xie, M. J. Yang, P. S. Chen, J. G. Restrepo, A. Karma, A. Garfinkel, Z. Qu, and J. N. Weiss, Biophys. J. 94, 392 (2008).

[16] J. Wagner and J. Keizer, Biophys. J. 67, 447 (1994).

[17] D. T. Gillespie, Annu. Rev. Phys. Chem. 58, 35 (2007).

[18] F. James, Comput. Phys. Commun. 60, 329 (1990); G. Marsaglia, A. Zaman, and W.-W. Tsang, Stat. Probab. Lett. 9, 35 (1990).

[19] M. Shang-Keng, Modern Theory of Critical Phenomenon (De Capo Westview Press, New York, 2000).

[20] H. Chaté and P. Manneville, Chaos 2, 307 (1992).

[21] R. H. Swendsen and J. S. Wang, Phys. Rev. Lett. 58, 86 (1987).

[22] P. Marcq, H. Chaté, and P. Manneville, Phys. Rev. E 55, 2606 (1997).

[23] K. Binder, Monte Carlo Methods in Statistical Physics (Springer-Verlag, Berlin, New York, 1979), Topics in current physics, 7.

[24] M. Nivala, C. Y. Ko, M. Nivala, J. N. Weiss, and Z. Qu, Biophys. J. 102, 2433 (2012).

[25] J. Miller and D. A. Huse, Phys. Rev. E 48, 2528 (1993).

[26] D. A. Egolf, Science 287, 101 (2000). 